\title{
The Research on the Construction of Teaching System Based on Fostering the Entrepreneurial Ability of College Students
}

\author{
(SUBMITTED BUT NOT PRESENTED)
}

\author{
XIONG Xiaoliang \\ Department of Economy and Commercial Foreign Language \\ Wuhan Technology and Business University \\ Wuahan City, Hubei Province, China \\ 345195568@qq.com
}

\author{
BEI Shu \\ Department of Economy and Commercial Foreign Language \\ Wuhan Technology and Business University \\ Wuahan City, Hubei Province, China \\ Susanshu1985@163.com
}

\begin{abstract}
In the era of knowledge-driven economy, with the transformation of economy and society, liberation of human being and the gradual popularization of modern information technology and network technology, the carrier of entrepreneurial knowledge becomes more and more various. Meanwhile,the entrepreneurial knowledge has been transferred and delivered in more various ways. As a result, the generation, accumulation, exchange and application management of knowledge change correspondingly. It becomes the basis and core of the cultivation of entrepreneurial ability to reconstruct the entrepreneurial context and reflect the entrepreneurial activity initatively. This paper proposes the strategy of constructing new entrepreneurial teaching system based on the analysis of entrepreneurial knowledge context, entrepreneurial knowledge supply, the generation and delievery of entrepreneurial knowledge demand and the flow of entrepreneurial knowledge in the internet community as well as the constitution and features of internet community.
\end{abstract}

Keywords-college education; entrepreneurial ability generation; self-development;teaching system

\section{INTRODUCTION}

In the era of knowledge-driven economy, with the transformation of economy and society, liberation of human being and the gradual popularization of modern information technology and network technology, the environment for the generation of entrepreneurial knowledge changes. The carrier of entrepreneurial knowledge becomes more and more various. Meanwhile,the entrepreneurial knowledge has been transferred and delivered in more various ways. As a result, the generation, accumulation, exchange and application management of knowledge change correspondingly.

To begin with, within the new economic system, the priority has been shifted to the exploitation of intelligence resources from the traditional production. Therefore, the flow of entrepreneurial knowledge could realize the capitalization and productization. The management of entrepreneurial knowledge generation attracts attention from the academic circle and business circle.

In the second place, with the sharp increase of entrepreneurial knowledge and information, and the wider application of computer and internet technology, people's ability of processing data has been promoted. Consequently, people can acquire the entrepreneurial knowledge from a larger scale, at a higher speed and lower cost.

Thirdly, there are various forms of reservation space for entrepreneurial knowledge, such as people's brain, group consciousness, paper carrier and digitized carrier, which has raised the efficiency of the utilization of knowledge and is helpful to the application of different knowledge.

Fourthly, entrepreneurial knowledge flows more quickly and precisely among different carriers in the information era. During the process, new entrepreneurial knowledge may be generated. Thus, the way of entrepreneurial knowledge generation becomes various. In a word, both the quality and quantity of entrepreneurial knowledge may be increased.

Fifthly, "human being" is the key factor for the reservation and delivery of entrepreneurial knowledge. Human resource is not only the important carrier of knowledge but also the subject of the generation of entrepreneurial knowledge. The generation of entrepreneurial knowledge is realized by people's brainwork The management of entrepreneurial knowledge generation extraordinarily calls for creative intelligence. Only in this way can the atmosphere and mechanism of entrepreneurial knowledge generation be developed.

Although modern technology has promoted the development of some aspects of entrepreneurial knowledge, it brings some obstacles to the generation of entrepreneurial knowledge. The first one is the redundancy of information and technology. With the wide application of information theory, there are more information symbols than the amount that is actually needed during the process of information delivery. Some knowledge is helpful for individuals to improve the quality and efficiency of their work but does not affect other 
individuals or the whole organization. The socialization and externalization of entrepreneurial knowledge rely on the communication among individuals. Without the interaction, the transformation of entrepreneurial knowledge may lose its source of energy. Meanwhile, the transformation of entrepreneurial knowledge also needs guidance which lies not only the collection and assembly of scattering entrepreneurial knowledge in different carriers but also the systematic training of workers. The second obstacle is the "created disorder". When the entrepreneurial knowledge community faces the same problems and the owners of different knowledge affect each other while solving the same problem, the collision and integration of different knowledge take place. The new knowledge generated by the "created disorder"is unique and different from previous entrepreneurial knowledge or knowledge owned by other enterprises. The environment that the generation of knowledge depends on can not be developed without the advisable and careful management of leaders. Meanwhile, the formation of collaborative and creative culture is also necessary. The third obstacle is the free will of the members of entrepreneurial teams. The free will can stimulate the initiative of members, promote them to dispaly their intelligence and encourage them to break the cognitive limitation and solve problems creatively. In order to avoid the conflicts caused by the convention and cognitive framework of members as well as ensure the active and constructive collaboration, developing the atmosphere of being cooperative is crucial to the generation of entrepreneurial knowledge.

\section{THE CHANGE OF ENTREPRENEURIALTEACHING MODALITY IN THE NETWORK COMMUNITY}

The integration of information, knowledge, and intelligence makes it possible not only to deliver entrepreneurial knowledge to people in time but also to make the application of generation, accumulation, exchange and application management of knowledge influence on different fields, which exists not only in the ivory tower of philosophers, psychologists and sociologists but also in the macroscopic knowledge management of a country as well as the microcosmic knowledge management of individual. In addition, it spreads to the comprehensive classroom of various information technology and different courses. Meanwhile, it has its own law of change and development. It has become an issue that can not be neglected to explore the organization and feature of prevalent internet communities as well as the flow and influence of entrepreneurial knowledge.

\section{A. The Demand of Entrepreneurial Knowledge being more dependent on the Context}

The realistic requirement of entrepreneurial knowledge is the starting point of modern pedagogy. Entrepreneurial knowledge refers to not only the static knowledge but also the dynamic flow of knowledge. As the knowledge flows among the members within certain organization, the demand for some knowledge is passed on to the creator. With the continuous deepening of information technology revolution and networking, the social environment changes fast, which brings uncertainty that stimulates new demand for entrepreneurial knowledge. Some forms of entrepreneurial knowledge exists in the brain of some people, while other forms of entrepreneurial knowledge exist in the business process and knowledge base. The influence of knowledge as well as its dependence on the context vary in different carriers. The traditional teaching relying on books involves the clear explanation of knowledge in the books, transformation of knowledge into the form that is easy for reader to understand or the systematization of fragmentary knowledge, which only meets the demand that is less dependent on the context. Therefore, teachers need to respond sensitively to the new entrepreneurial knowledge and acquire them to make use of them in the classroom teaching. The demand for entrepreneurial knowledge which is very dependent on the context is implicit. The key lies in the individual's acquisition of entrepreneurial knowledge from others through observation, imitation and communication by some technological instruments, such as new network sites, wiki, micro blog and so on. In this way, the explicit knowledge from the internet communities can be transformed into the implicit individual knowledge.

\section{B. The Teaching Field of the Entrepreneurial Knowledge Extending}

Teaching field is not only the environment of teaching but also the space for teachers to construct the entrepreneurial knowledge continuously. The environment of individual student will be transformed into their own entrepreneurial environment through the filtration of teacher's experiences and present reservation of entrepreneurial knowledge. Although teacher's classroom and office are relatively quiet and there are no distraction, which may be helpful to arouse the creativity of both teachers and students, it is may be quite difficult for teachers and students to make any real achievement due to lack of exchange of information and energy. With the development of multimedia teaching and internet teaching, teaching activity becomes increasingly open. Teachers need to broaden their vision to make use of any entrepreneurial teaching resources for their classroom teaching while they prepare for their lecture. Teachers also need to break the limitation brought by the traditional perception of teaching to take the students' creativity into consideration and realize the effective communication on creativity between teachers and students. In addition, at the stage of teaching feedback, teachers need to adjust the schedule and speed of teaching to make appropriate decision and enter new world of entrepreneurial knowledge.

\section{Deconstructing Teachers' Authority of Knowledge}

Since the class-based teaching system was proposed by Comenius who is a Czechic educator, the mode of teachers' existence seems to have been fixed. Teachers live for teaching which is a regular and predictable process.Five Formal Steps proposed by Herbat has always been considered to be the classic theory. Based on the fixed social position, moral expectation and recognition of role, teachers have clear perception of their own career and the role they play. They also have recognition of their role. However, the fast development of internet technology makes it possible to spread information widely and acquire knowledge conveniently. Thus, cultural feedback becomes increasingly obvious. Meanwhile, the authority of teacher's knowledge is 
challenged. Teacher's role and identity become obscure and splitting, which give birth to teacher's existential anxiety, trust crisis , ontological security threats and some other problems. Fortunately, teacher's identity crisis is also the opportunity for teachers to awake their self-awareness. The generation of entrepreneurial knowledge needs this kind of awareness. Teacher's consciousness of being the subject can help teachers accept new knowledge, update their knowledge structure, reflect their teaching process and make their thinking active all the time. All of these offer wider space for the generation of entrepreneurial knowledge.

\section{Constructing The Network of Entrepreneurial Knowledge}

Entrepreneurial teaching activity which involves different subjects and many factors is dominated,constructed and guided by related knowledge. In other words, entrepreneurial teaching activity is related with many other factors both internally and externally. In the traditional "teaching-accepting"mode, teacher is the authoritative owner of entrepreneurial knowledge. Their sole duty is to teach and deliver knowledge to students. They stick to the center of the classroom, center of the subject, center of the school. There are only some exploitation and drilling on the ability of recitation, comprehension and analysis. There is lack of exchange of knowledge and wisdom, lack of communication on the spirit and meaning as well as lack of the sharing of knowledge and experience.

Luckily, the development of information technology and spread of the spirit of internet bring us new technology, new knowledge and various creativity. Besides, they trigger the collision and fission of many cultural factors in school. The learning community not only concerns the delivery of knowledge but also the exchange upon common topic. Teachers begin to admit their confusion they meet when they try new teaching method and invite others to visit as well as discuss their own teaching world. There are sincere communication about each other's feeling and suggestion among teachers. At the same time, students start to challenge the knowledge and authority of education. Teachers begin to transfer from the leading role of education to the chief between the equal relation with students. During this process, teachers and students share their thought, experience and knowledge and exchange each other's feeling and opinions to achieve mutual progress. Finally, there will be a knowledge network community which includes teachers, students and knowledge.

\section{CONSTRUCTING THE ENTREPRENEURIAL TEACHING SYSTEM BASED ON THE GENERATION OF ENTREPRENEURIAL ABILITY}

The evolution of the environment for the generation of knowledge and the modality of the generation of knowledge lead to the reconstruction of knowledge and reconstruction of courses. While colleges continue to place emphasis on the basic knowledge and basic ability in the traditional courses, they increase the number of courses of entrepreneurial practice and the entrepreneurial activities. The following content is some suggestion about how to construct the practical teaching system based on the probe of mutual influence between the subject and external structure of entrepreneurial knowledge generation.

\section{A. "Knowledge--Attitude" Oriented Teaching Purpose}

With regard to the professional education in college, the education of knowledge is crucial. Students'grasp of knowledge that is both the reason and purpose of teaching is also the achievement that teachers apply various teaching approaches and organize different teaching activities to make. To some extent, it is also the standard of inspecting and assessing teaching activities. At present, the knowledge that students need to master is decided by their major in college. Among the knowledge, theoretic knowledge and academic knowledge are very important for students to learn. Therefore, the value of teaching lies in the delivery of knowledge and scientific achievement. Individual experience and feeling are considered to be unreasonable and not taken into consideration.

But it should not be neglected that the generation and construction of entrepreneurial knowledge is the result of the interaction between human being and environment in the complex context. Besides, students are the active constructor of knowledge system. Thus, the development of human should always be the ultimate purpose of education. Among the different aspects of human's development, the cultivation of creativity should be given priority.

Another important aspect is the cultivation of students' proper attitude. Guided by this purpose, education may concern students' feeling and experience of creativity, and lead students to care and understand themselves, other people, nature and society through caring themselves, participating activities and solving problems. Students'proper attitude is formed and developed during the continuous interaction with teachers, students and courses. It concerns the comprehensive growth of students rather than how much knowledge they have copied.

To cultivate students' proper attitude, educators should pay special attention to the following two aspects. Firstly, students'interest in creativity as well as the motivation and passion of creativity need to be stimulated to develop their attitude of being fond of creativity. Secondly, since education not only concerns the students' grasp of knowledge but also needs to guide students to have better understanding of the life, educators should guide students to develop positive understanding of starting business which includes their optimistic attitude toward life, realistic attitude toward science and tolerant attitude toward others. To achieve this purpose, students should be guided to understand the unification of individual value and social value as well as the unification of scientific value and humanistic value during the classroom teaching.

\section{B. “Text--Knowledge” Oriented Teaching Content}

Traditional entrepreneurial teaching has its own disadvantages. At the beginning, traditional entrepreneurial teaching is usually based on classic books or textbooks chosen by professional experts. As a result, traditional teaching process is simplified into some fixed teaching steps. To some extent, it is essentially the reflection of teaching plan. But it should not be forgotten that understanding the teaching content should be based on the understanding of teaching objective, the exploitation of the relation between the teaching objective and the text books and comprehending the teaching process. It is 
obvious that the planned teaching process lacks flexibility and vitality. The initiative ability, reflective ability and creativity can not be practiced or developed.

Actually, individuals may choose some of the new knowledge to absorb and construct their own structure of entrepreneurial knowledge. Teachers and students need to make decision and integration continuously in certain entrepreneurial context to form their practical knowledge.

Proper entrepreneurial context is crucial to the entrepreneurial teaching. Teachers should establish the proper entrepreneurial context which is the platform used to present entrepreneurial knowledge and for the collective study. Usually, this kind of entrepreneurial context should meet the following demand. Firstly, it must be related tightly with students'previous knowledge and experiences. It should arouse students' interest in new knowledge. But at the same time, it should not surpass students' present cognitive ability too much. Only when the entrepreneurial knowledge and entrepreneurial context are defined and understood by students, can students learn knowledge initiatively. Secondly, teachers should make use of the computer and internet to establish the virtual collaborative context. In this way, students can have virtual experience of what they have learned in the classroom. It is also convenient for teachers to adjust their learning and encourage them to learn continuously through the exchange with them on the internet. Thirdly, the internet can be connected with successful classroom teaching. The real entrepreneurial cases can be collected to establish the distinctive case base. As a result, entrepreneurial teaching and teaching reform in college take place simultaneously.

\section{C. “Acceptance--Communication" Oriented Teaching Design}

In the present structure of classroom teaching, teachers and students are isolated from each other. The relationship among them seems to be conservative. Accordingly, teachers' teaching philosophy and teaching activity are characterized by autarchy, discipline, ranking and denial.Teachers, standing on the platform, stress on the delivery of theoretic and academic knowledge and the teaching process of deduction, but ignore the experienced inductive process of teaching. During the process, teachers observe, get to know, analyze and classify students in order to manage and control them better as well as realize the expectation. At the same time, students consider teachers to be the deliverer of knowledge whom they can acquire knowledge from and the technician who can help them to achieve their learning aim. Consequently, both teachers and students can hardly experience the passion, energy, curiosity and joy brought by teaching and learning.

Actually, there should be communication on starting business in addition to the delivery of knowledge between teachers and students. Teachers transform into the role of chief entrepreneur. Teachers and students exchange their opinions on creativity and originality, share their entrepreneurial knowledge and experience and appreciate the meaning and value of life together.

When teachers design and organize their classroom teaching, it is crucial for the transformation of the relationship between teachers and students that the environment in which entrepreneurial knowledge can be acquired, created, applied and accumulated repeatedly is created. This environment is essentially a system that can be divided into three layers. On the top layer is the project team. Project teams conduct thematic study or subject study on the real problems or issues with comprehensive knowledge of different subjects or the knowledge of one subject. On the second layer is the platform of organizational system for entrepreneurial probation or entrepreneurial internship, through which teachers and students can participate in the companies's normal and routine businesses. At the bottom layer is the knowledge base of entrepreneurial courses in which the knowledge generated by the project teams'work and probation as well as the internship is collected and classified.

In the system, project teams create the entrepreneurial knowledge through their practical study and socialization and generate notional knowledge through externalization. Then, those knowledge is transferred to the bottom layer to be analyzed, classified, reserved and transferred to the middle layer to become students' operational knowledge through internalization to support daily work and practice of enterprise. During this process, the knowledge is inserted into the structure. Then, it may be transferred to the new project team and the circulatory system of knowledge is formed.

\section{D. "Isolation--Communication" Oriented Teaching Culture}

Teaching culture guides the teaching activity through teaching value organization. The teaching designing, teaching method, teaching strategy and interpersonal relationship during the teaching process are connected with teacher's values and thinking mode in various ways. The closure of teaching space and the repetition of teaching activities result in the fact that teachers may complete their teaching according to the prearranged content in the teaching reference books without any reflection or critical thinking. Although there are many rules in the school which are designed to ensure the quality of teachers' teaching, such as collective lesson preparation, attending and assessing lectures, teachers may be accustomed to their individual teaching habit which is hard to be changed. Therefore, there will be few collaboration among teachers and isolated values, thinking mode and behavioral habit.

But it can not be neglected that the collaborative teaching culture is meaningful to entrepreneurial teaching. It will be helpful to form the similar values and collaboration among teachers which may give birth to the trust among teachers that is the base of the exchange of knowledge of innovation and entrepreneurship among teachers. Hence, it is significant for us to develop the atmosphere of mutual trust, break the isolation among teachers, change teacher's attitude of being ordinary and improve the entrepreneurial practical ability with the collaborative work. Besides, we need to construct an open entrepreneurial practical environment where there is challenge but no threat. In this environment, teachers encourage students to be creative, accept failure and summarize the lessons to share with others. 


\section{REFERENCES}

[1] Mingsheng Zhang, Houqing Yin, and Yi Yu, eachers Humanities Reading. Shanghai: Shanghai Lexicographical Publishing House,2013. J. Clerk Maxwell, A Treatise on Electricity and Magnetism, 3rd ed., vol. 2. Oxford: Clarendon, 1892, pp.68-73.

[2] Fuller, Sociology of Knowledge and Life. Trans1. Xiaoting Jiao. Beijing: Peking University Press,2011.

[3] Giroux, Teachers as Intellectuals. Trans1. Hongwen Zhu. Beijing: Education and Science Press, 2008K.
[4] Andy Hargreaves, The Teaching in Knowledge Society. Shanghai: East China Normal University Press, 2007.

[5] Erxun Cai, "Study on the Orientation of Teacher's Role and Function Under the U-Learning Mode", in Journal of Fujian Radio and TV University, vol. 4. Fujian, 2011.

[6] Hongjian Pan, "Study on the Generation of Individual Knowledge During the Curriculum Implementation" ", in Curriculum Textbook Teaching, vol. 2007 